\title{
VIII.
}

\section{Zur Casuistik der traumatischen Erkrankungen des Gehörorgans.}

Fon

F. Trautmann.

1. Hämorrhagien im Trommelfell nach einem einmaligen Hustenanfall.

In der Nacht des 31. Mai 1877 erkrankte um 121/2 Uhr Joseph B., 21 Jahre alt, während er im Bette schlief, plötzlich am rechten Ohre und zwar in Folge eines Hustenanfalles. $\mathbf{E r}$ hatte sich vollständig gesund zu Bett gelegt und vorher weder an den Ohren noch Lungen gelitten. Er gab an, nach dem Hustenanfall rechts plötzlich hochgradig schwerhörig geworden zu sein, so dass er seine Cylinderubr, welche er sofort an das rechte Ohr legte, nicht mehr hören konnte; ausserdem empfand er Kopfschmerzen, ein continuirliches Sausen im rechten $\mathrm{Ohr}$ und ein taubes Gefühl in der rechten Gesichtshälfte; im Ohre selbst empfand er geringe Schmerzen, die nur kurze Zeit anhielten. Sein Allgemeinbefinden war durch diese Erkrankung nicht gestört. Schwindel, Uebelkeit, Erbrechen sind nicht vorhanden gewesen.

Die Untersuchung am 1. Juni 1877 ergab Folgendes: Mässig kräftiger Körperbau, gut entwickelte, straffe Musculatur, Fettpolster gering, Bindehaut der Augen, Schleimhaut des Mundes und der Nasenrachenhöhle normal, frei von Blutextravasaten; Herz normal; die Lungen ergeben keinen Anhaltspunkt für den Hustenanfall, so dass wahrscheinlich während des Schlafes, der vielleicht mit geöffnetem Munde stattfand, ein Fremdkörper (Insekt?) in den Keblkopfeingang gelangte und den Hustenparoxysmus veranlasste. Lähmungserseheinungen waren nicht vorhanden. 
Knochenleitung fur Uhr rechts angeblich geschwunden, links erhalten, Stimmgabelversuche vergessen zu notiren, Uhr wird rechts an die Ohrmuschel gelegt, nicht vernommen, Flïstersprache wird rechts angeblich nicht gehört, links ist die Hörfähigkeit für Uhr und Sprache normal. Aeusserer Gehörgang weit, zeigt keine Abnormität, Trommelfell rechts leicht diffus getrübt, deshalb nicht mehr von normaler Transparenz, Hammer normal gestellt, Gefässe desselben injicirt, dreieckiger Lichtreflex von normaler Form, sein Glanz vermindert. Im vorderen Abschnitt des Trommelfells befinden sich vier, etwa stecknadelkopfgrosse Hämorrhagien, jedoch nicht vollkommen rund, sondern etwas zackig, drei befinden sich oberhalb des dreieckigen Lichtreflexes, eine unterhalb desselben und ist letztere die grösste. Im hinteren oberen Quadranten, dicht an der Peripherie, sieht man eine sichelförmige Hämorrhagie, etwa $3 \mathrm{Mm}$. lang, $1 \mathrm{Mm}$. breit, zwei stecknadelspitzengrosse an der hinteren Hammerkante, die eine dicht unter dem kurzen Fortsatz, die andere am Ende des Hammergriffes. Bei $+V$ und $-\mathrm{S}$ bewegt sich das Trommelfell normal, ein freier Bluterguss in der Paukenhöhle ist nicht vorhanden, Hämorrhagien in der Paukenschleimhaut lassen sich durch das nicht vollständig transparente Trommelfell nicht erkennen. Die Hämorrhagien im Trommelfell erheben, wie man mit dem Vergrösserungstrichter sieht, leicht die Epidermis uber die Oberfläche, haben demnach nicht ihren Sitz in der Schleimhaut, sondern in der Dermisschicht. Links Trommelfell normal zu nennen. Der Nasenrachenraum ist weit, ebenso der untere Nasengang. Tuba für den Katheter leicht zugängig, rechts jedoch leichter als links. Auseultationsgeräusch weich und breit.

Die Kopfschmerzen waren nach 24 Stunden vollständig geschwunden, das Gefühl von Taubheit nach 4 Tagen, das Sausen nach 9 Tagen. Die Knochenleitung kehrte nach 5 Tagen zuriick und wurde die Stimmgabel zu dieser Zeit angeblich beiderseits gehört, obgleich die Hämorrhagien im Trommelfell noch vorhanden waren.

Die Hörfähigkeit besserte sich täglich und war nach drei Wochen zur Norm zurïckgekehrt. Die Hämorrhagien wanderten nach der Peripherie des Trommelfells und dem äusseren Gehörgang und hatten nach 20 Tagen noch eine schmutzigbraune Farbe.

Die Behandlung bestand im Fernhalten von Schädlichkeiten (nicht Schnaufen, Tragen ron Watte im äusseren Gehörgang), 
Zur Casuistik der traumatischen Erkrankungen des Gehörorgans, 115

ferner in localer Blutentziehung dicht vor dem Tràgus, Magn. sulf. innerlich.

\section{Doppolfissur des Trommelfelis links nach Ohrfeige,}

Heinrich W., 22 Jahre alt, erhielt am 12. Januar 1878 eine Ohrfeige gegen das linke $\mathrm{Ohr}$, empfand sofort Sausen, ziemlich lebhafte Schmerzen und Schwerhörigkeit in dem betreffenden Ohre. Die Schmerzen liessen allmählich nach, Sausen und Schwerhörigkeit bestanden angeblich noch bei der Untersuchung am 13. Januar 1878, 'wo sich Folgendes ergab:

Ohrmuschel und Umgebung normal, bei Druek nirgends Schmerzen. Nase und Nasenrachenraum zeigen keine Abnormitäten. Rechts: Trommelfell normal, Glanz etwas abgeschwächt. Links: parallel hinter dem Hammergriff, etwa $1 \mathrm{Mm}$. von demselben entfernt eine Fissur, die $1 \mathrm{Mm}$. unter dem kurzen Fortsatz beginnt und bis zum Ende des Hammergriffes zieht. Im hintern unteren Quadranten Blutcoagulum. Im vordern unteren Quadranten eine Narbe etwa erbsengross, die hintere Peripherie der Narbe eingerissen. Bei $+V$ dringt die Luft durch diesen Riss, sowie durch die Fissur hinter dem Hammergriff. Stimmgabel wurde links-gehört. Knochenleitung beiderseits fü Uhr vorhanden. Uhr rechts 4 Fuss, links angeblich angelegt nicht; zugewandte Flüstersprache rechts angeblich nur 10 Fuss, links wird Flüstersprache angeblich nicht gehört. Jedenfalls liegt Uebertreibung vor. Bei der Entlassung am 26. Februar hatte sich die Hörfähiglreit wesentlich gebessert, wenn auch immer noch uibertrieben wurde. Zugewandte Flüstersprache links 6 Fuss, Uhr 1 Zoll. Die Heilung fand nicht per primam, sondern durch geringe Eiterung statt, weil das Ohr durch das Versehen eines Heilgehülfen ausgespritzt worden war.

Narben werden durch Obrfeigen ziemlich häufig zerrissen, dass sich aber gleichzeitig noch eine Fissur im Trommelfell vorfindet, wie im vorstehenden Falle, dürfte zu den grössten Selten: heiten gehören, weshalb ich vorstehenden Fall, der sonst nichts Aussergewöhnliches bietet, mittheile.

3. Geheilte Fissur der Pyramide des Felsenbeines, der oberen Wand des knöchernen Gehörganges und des Trommelfelles links.

Otto M., 22 Jahre alt, seit dem 8. Juni 1877 wegen einer Kopfverletzung behandelt, wurde mir am 22. Juni wegen Ohren- 
sausen und Taubheit links vorgestellt und ging am 28. August in meine Behandlung über.

Der pp. M. wollte am 8. Juni 1877 Abends 6 Uhr eine Protze aus der Schmiede mit einem Kameraden nach der Kaserne fahren. Er nahm die Deichsel auf die linke Schulter und sein Kamerad schob hinten an dem Protzkasten. Beim Umbiegen um eine Strassenecke flog die Deichsel dem pp. M. gegen das linke Scheitelbein und verletzte die Weichtheile. Die Blutung aus der Wunde war nicht so bedeutend, als die aus dem linken äusseren Gehörgang. Ausserdem hatte er Nasenbluten, welches jedoch bald aufhörte. Er fiel bewusstlos zur Erde, wurde durch 2 Mann nach der einige Minuten entfernten Kaserne und dann mittelst einer Droschke sofort nach dem Lazareth gebracht. Die Bewusstlosigkeit dauerte nach Angabe des Arztes, welcher den pp. M. zuerst untersuchte, 5 Minuten, die Wunde auf dem linken Scheitelbeine ging bis auf den Knochen, das Periost soll nicht verletzt gewesen sein. Die Blutung aus dem äusseren Gehörgange war so bedeutend, dass sie tamponirt werden musste. Die Wunde wurde antiseptiseh verbunden. Erbrechen ist nicht vorhanden gewesen, Störungen in der Sensibilität und Motilität fehlten. Der Puls, welcher am Abend des 8. Juni 58 betrug, aber regelmässig war, stieg den nächsten Tag auf 80, ging aber dann auf 60 herab und blieb constant später 76 . Temperatursteigerung soll er nicht gehabt haben. Der in den ersten zwei Tagen retardirte Stublgang wurde vom dritten Tage an regelmässig. Die Wunde heilte unter dem Lister'schen Verbande per primam, der Charpiepfropf wurde nach zwei Tagen aus dem äusseren Gehörgang entfernt, ohne dass die Blutung wiederkehrte, nur hatte sich nach zwei Tagen der Charpiepfropf mit blutig seröser Flïssigkeit imprägnirt, und floss nach der Entfernung desselben entschieden Liquor cerebrospinalis aus dem äusseren Gehörgange. Sofort nach der Verletzung, als der pp. M. wieder bei Bewusstsein war, wurde festgestellt, dass bei $+V$ die Luft durch das Trommelfell piff. Bei seiner Aufnahme im Lazareth am 6. Juni 1877 klagte er iiber Sausen links und Taubheit. M. will früher anch links ganz gut gehört haben und an den Ohren niemals krank gewesen sein. Er klagt anch heute, am 22. Juni noch über Ohrensausen und Taubbeit links, das Sausen wird als ein continuirliches beschrieben, in die Tiefe des Ohres verlegt und dem Dampf der Locomotive verglichen, Die Untersuchung am 22. Juni 1877 ergibt Folgendes: 
Auf dem linken Scheitelbeine, $10 \mathrm{Cm}$. senkrecht nach aufwärts vom Tragus findet sich eine frische Narbe, welche im sagittalen Durchmesser von vorn nach hinten verläuft, $3^{1 / 2} \mathrm{Cm}$. lang, $2 \mathrm{Mm}$. breit und nicht mit dem Knochen verwachsen ist. Die sorgsame Untersuchung des Schädels ergibt den Knochen nirgends empfindlich, nirgends eine Callus-Wucherung, welche vielleicht auf Fissur deuten liesse; vor allen Dingen ist dies nicht der Fall im Bereiche des linken Scheitelbeines und der Schuppe des linken Schläfenbeines. Die Lymphdrüsen auf dem Processus mast. links sind geschwollen, die ubrigen in der Umgebung des Ohres sind nicht geschwollen, die Weichtheile uber dem Processus mast. vollständig normal.

Ein leiser Druck auf den linken Processus mast. ist nicht empfindlich, druckt man aber intensiver auf denselben und zwar nach der Frontalebene zu, so empfindet M. lebhafte Schmerzen, Schwindelgefühl und das Sausen steigert sich. Störungen in der Sensibilität oder Motilität sind nirgends vorhanden. Das Gebiet des Facialis vor allen Dingen ist vollständig intact.

Die Cylinderuhr ( 7 Fuss normal) wird links weder von der Schuppe des Schläfebeines, noch vom Processus mast. gehört, rechts wird die Uhr von dem betreffenden Knochen gut gehört. Die Stimmgabel wird von Scheitel, Stirnbeinen und den Zähnen nur rechts gehört, selbst wenn der Finger in den linken äusseren Gehörgang gesteckt wird. Weder für hohe noch für tiefe Tö̀ne noch für Geräusche ist das linke Ohr empfindlich, der Kranke hört den Resonanzton der $\mathrm{C}^{\prime}$-Stimmgabel nur rechts. Rechts Uhr angeblich nur 4 Zoll, zugewandte Fluistersprache 10 Fuss, links wird die Uhr nicht gehört, zugewandte Flüstersprache nicht, durch das Hörrohr gesprochene Worte ebenfalls nicht, d. h. nur mit Fliustersprache, da sie sonst ja durch die Knochenleitung mit dem anderen Ohre gehört werden. Trommelfell rechts in der Peripherie getrübt, hintere Falte deutlicher als normal, Hammer normal gestellt, gelber Fleck normal, dreieckiger Lichtreflex fehlt, dicht vor dem kurzen Fortsatz ein feiner Lichtpunkt, Hammergefässe mässig injicirt, bei $+V$ bewegt sich das Trommelfell normal. Lin $\mathrm{ks}$ zeigt das Trommelfell im hinteren oberen Quadranten ein Blutcoagulum, welches den kurzen Fortsatz und das obere Drittel des Hammergriffes verdeckt. Es wird vorsichtig mit der Spritze entfernt und-ergibt die Untersuchung darnach das Trommelfell ebenfalls in der Peripherie getrübt, nur etwas stärker als rechts; dicht an der hinteren Kante des Ham- 
mergriffes sieht man eine dentliche, sehr feine, aber stark geröthete Narbe, die vom kurzen Fortsatz beginnt und $3 \frac{1}{2} \mathrm{Mm}$. nach unten zieht, rom kurzen Fortsatz zieht sie $2 \mathrm{Mm}$. nach oben und dann etwa $4 \mathrm{Mm}$. nach hinten und oben, so dass sie gezackt wird.

Bei $+V$ bewegt sich das Trommelfell deutlich, die Narbe röthet sich noch intensiver, aber es dringt keine Spur von Luft mehr durch, auch beim Katheterisiren ist kein Perforationsgeräusch za hören. Durch das Trommelfell ist ein Bluterguss in die Paukenhöhle nicht zu constatiren, auch ist das Auscultationsgeräusch normal und deshalb ebenfalls ein Bluterguss auszuschliessen. Nach Katheter keine Veränderung in der Hörfähigkeit oder im subjectiven Geräusche.

Die Untersuchung des Nasenrachenraumes ergibt denselben normal mit Ausnahme einer mässigen Schwellung der Rachentonsille an der 'linken Hälfte der Schädelbasis. Dieser' Theil der' Schädelbasis ist auch bei der Untersuchung mit dem Finger schmerzhaft und gleichzeitig tritt Schwindelgefithl und Steigerung: des Sausens ein.

Die Blutungen aus dem äusseren Gehorgange sind in diagnostischen Beziehungen äusserst werthroll. Sobald die Blutung: so gross ist, dass sie aus dem äusseren Gehörgange heraustritt, kann man mit Sicherheit annehmen, dass die Verletzung nicht nur das Trommelfell betroffen hat, sondern entweder den Knochen oder das Mittelohr. Durch Sturz oder Schlag gegen das Kinn fracturirt nicht selten die hintere Wand der Gelenkgrube des Unterkiefers, welche gleichzeitig die vordere Wand des knöchernen Gehörganges bildet; dabei kann es zur Fissur des Trommelfells im vorderen Abschnitte kommen. Bei Fissuren der hinteren oberen Wand des knöchernen Gehörganges, welche immer mit Fissur der Schädelbasis verbunden sind, findet eine Fissur des Trommelfelles im hinteren oberen Quadranten statt und die Blutung ist stärker als bei der Fractur der vorderen Wand des. knöchernen Gehörganges. Bei Fissuren des Trommelfelles ohne Fractur ist die Blutung äusserst unbedeutend und beträgt nur einige Tropfen, welche auf der Fissurstelle selbst antrocknen. Von den Blutungen bei polypösen Wucherungen sehe ich ab, da es sich hier nur um Blutungen bei normalen Verhältnissen handelt. Die Diagnose der Fissur des linken Felsenbeines wird unterstiitzt durch die sehr bedeutende Blutung áus dem äusseren. Gehörgange, stattgehabte Bewusstlosigkeit, fortwährenden Schwin- 
del nach der kranken Seite hin, continuirliches Sausen, absolute Taubheit, Steigerung des Schwindels durch Verschiebung der Knochenfragmente vom Processus mast. und dem Nasenrachenraum und zwar nur von der entsprechenden Hälfte der Schädelbasis aus in der Frontalebene, Abfliessen von Liquor cerebrospinalis aus dem äusseren Gehörgange.

Die Behandlung bestand in kalten Umschlägen auf Processus mast. und Stirn, localer Blutentziehung, Abführmitteln, Ruhe, Regelung der Diät, später Jodkali. M. blieb fieberfrei, sobald er jedoch versuchte, und zwar erst nach 6 Wochen, das Bett zu verlassen, steigerte sich der Schwindel, welcher immer nach der linken Seite gerichtet war, die subjectiven Geränsche wurden sehr intensiv, ausserdem trat hohes Fieber ein, welches immer einige Tage anhielt. Erst 6 Monate nach der Verletzung kann M. dawernd ausserhalb des Bettes weilen; ist jedoeh noch nicht im Stande, schnell zu gehen oder sich körperlich anzustrengen, ohne dass Schwindel mit der Richtung nach links eintritt. Druck auf Processus mast. und auf die linke Hälfte der Schädelbasis (Digitaluntersuchung des Nasenrachenraumes) ruft selbst nach Verlauf von 7 Monaten sofort Schwindelgefühl und gesteigerte Geräusche hervor, woraus hervorgeht, dass die Heilung der Knochenfragmente wobl mehr durch fibröses als dureh Knochengewebe stattgefunden hat.

Links besteht auch jetzt noch absolute Taubheit verbunden mit subjectivem Geräusch. Der Appetit ist ungestört, Schlaf ruhig, Körperernährung hat nicht gelitten. Lähmungserscheinungen sind nicht eingetreten. $\mathrm{Ob}$ die Heilung eine dauernde bleibt, muss die Zeit lehren. 